\title{
Assessment of Global and Local Alterations in Retinal Layer Thickness in Ins2 (Akita) Diabetic Mice by Spectral Domain Optical Coherence Tomography
}

\author{
Andrew W. Francis, ${ }^{1}$ Justin Wanek $\mathbb{D D}^{2},{ }^{2}$ and Mahnaz Shahidi $\mathbb{D D}^{3}$ \\ ${ }^{1}$ Department of Ophthalmology, University of California San Francisco, San Francisco, CA, USA \\ ${ }^{2}$ Department of Ophthalmology and Visual Sciences, University of Illinois at Chicago, Chicago, IL, USA \\ ${ }^{3}$ Department of Ophthalmology, University of Southern California, Los Angeles, CA, USA \\ Correspondence should be addressed to Mahnaz Shahidi; mshahidi@usc.edu
}

Received 9 October 2017; Accepted 24 December 2017; Published 20 February 2018

Academic Editor: Lawrence S. Morse

Copyright $\odot 2018$ Andrew W. Francis et al. This is an open access article distributed under the Creative Commons Attribution License, which permits unrestricted use, distribution, and reproduction in any medium, provided the original work is properly cited.

\begin{abstract}
Purpose/Aim. The Ins2 (Akita) mouse is a spontaneous diabetic mouse model with a heterozygous mutation in the insulin 2 gene that results in sustained hyperglycemia. The purpose of the study was to assess global and local retinal layer thickness alterations in Akita mice by analysis of spectral domain optical coherence tomography (SD-OCT) images. Materials and Methods. SD-OCT imaging was performed in Akita and wild-type mice at 12 and 24 weeks of age. Inner retinal thickness (IRT), outer retinal thickness (ORT), total retinal thickness (TRT), and photoreceptor outer segment length (OSL) were measured. Mean global thickness values were compared between Akita and wild-type mice. Local thickness variations in Akita mice were assessed based on normative values in wild-type mice. Results. Akita mice had higher blood glucose levels and lower body weights $(p<0.001)$. On average, IRT, ORT, and TRT were approximately $2 \%$ lower in Akita mice than in wild-type mice $(p \leq 0.02)$. In Akita mice, the percent difference between retinal areas with thickness below and above normative values for IRT, ORT, and TRT was $22 \%$, $32 \%$, and $38 \%$, respectively. Conclusions. These findings support the use of the Akita mouse model to study the retinal neurodegenerative effects of hyperglycemia.
\end{abstract}

\section{Introduction}

Diabetic retinopathy (DR) is one of the most common causes of blindness worldwide [1-4]. The pathophysiology of this condition remains poorly understood, but its development and progression are associated with uncontrolled hyperglycemia, inflammation, neuronal dysfunction, and hypoxia. These various elements ultimately lead to the upregulation of growth factors, endothelial cell-specific mitogens, proteinases, and cytokines that cause pathologic alterations in the retinal architecture [5-9]. The application of experimental rodent models has improved our understanding of the disease process in DR, though there remains a need for rodent models that can replicate both the early and late clinical manifestations of DR [5-30]. Although transgenic, knockout, and toxin-induced rodent models have been studied, no rodent model to date has been able to accurately reproduce all vision-threatening features of DR including neovascularization, cystoid macular edema, and ischemia [25, 31-45].

Spectral domain optical coherence tomography (SDOCT) imaging has become the standard of care in the diagnosis and management of DR [46-61]. Importantly, diabetic patients with minimal or no retinopathy demonstrate neurodegenerative alterations including retinal and choroidal thinning in SD-OCT images that often precede clinically identifiable DR $[12,13,16,17,20,21,49,50,62-66]$. It is of interest to investigate if retinal thickness alterations in patients with minimal to no DR can be reproduced in rodent models of diabetes. 
The Ins2 (Akita) mouse is a spontaneous hyperglycemic mouse model of diabetes with an insulin 2 protein gene mutation that results in pancreatic $\beta$-islet cell death, hypoinsulinemia, and hyperglycemia starting at approximately four weeks of age $[10,36,39,42,67]$. Han et al. showed that the Akita mouse was similar in all aspects to the wild-type mouse before hyperglycemia occurred at approximately 4 weeks of life [36]. In their work, no differences between Akita and wild-type mice in vascular area, layer thickness on histology, or neurodegeneration were noted at 4 weeks. Significant increases in apoptotic retinal cells were observed in Akita mice as compared to wild-type mice at 6 and 9 months of age.

The majority of research studies investigating retinal alterations in the Akita mouse have employed ex vivo techniques such as histological sectioning, immunofluorescence, and trypsin digest models or in vivo methods including fluorescein angiography and confocal microscopy $[10,36,37,39,42,43,68,69]$. There are a limited number of studies that have investigated retinal layer thickness alterations in the Akita mouse using SD-OCT imaging; however, the findings have been inconclusive. Specifically, retinal thickness was reported to be similar between Akita and wild-type mice by evaluating retinal thickness at localized regions using commercial software designed for human image analysis $[37,70]$. Another study found inner and outer retinal layer thinning in Akita mice by using a manual image segmentation method [33]. In the present study, we assessed both global and local thickness changes due to sustained hyperglycemia in the Akita mouse model of diabetes by SD-OCT volume imaging and applying a semiautomated image segmentation method to map thickness of individual retinal cell layers.

\section{Materials and Methods}

2.1. Animals. Imaging was performed in aged-matched diabetic Akita $(N=22)$ and C57BL/6J wild-type $(N=22)$ mice at 12 weeks (Akita: $N=11$, wild-type: $N=12$ ) and 24 weeks (Akita: $N=11$, wild-type: $N=10$ ) of age. The mice were treated in compliance with the ARVO Statement for the Use of Animals in Ophthalmic and Vision Research. The mice were fed a Teklad diet (http://www.envigo.com/resources/ data-sheets/7012-datasheet-0915.pdf), were exposed to $14 \mathrm{hr} / 10 \mathrm{hr}$ of light/dark cycle, and housed in cages with a maximum number of 5 mice per cage. The mice were then anesthetized with intraperitoneal injections of ketamine $(100 \mathrm{mg} / \mathrm{kg})$ and xylazine $(5 \mathrm{mg} / \mathrm{kg})$. Additional injections were given to sustain anesthesia as needed. On the day of imaging, nonfasting blood glucose levels were measured from a drop of blood obtained by tail puncture with a commercially available blood glucometer (FreeStyle Lite, Abbott, Alameda, CA). Body weights of the mice were obtained. The femoral artery of each mouse was cannulated, and a catheter was attached. The mice were then placed in an animal holder, and their pupils were dilated with $2.5 \%$ phenylephrine and $1 \%$ tropicamide. Prior to SD-OCT imaging, $10 \%$ fluorescein sodium ( $5 \mathrm{mg} / \mathrm{kg}$, AK-FLUOR ${ }^{\circledR}$, Akorn, Decatur, IL) was administered through the femoral artery catheter for fluorescein angiography. SD-OCT image data was acquired in one eye in each mouse (left: $N=14$; right: $N=30$ ). During imaging, eyes were kept hydrated by frequent administration of eye drops. Image data collected from left eyes were transformed to orient all data to a right eye configuration.

2.2. Image Acquisition. A SD-OCT raster scan was obtained using a commercially available instrument (Spectralis; Heidelberg Engineering, Heidelberg, Germany). The raster scan consisted of 31 horizontal B-scans with a depth resolution of $3.9 \mu \mathrm{m}$ and $1536 \mathrm{~A}$-scans per B-scan. The raster scan was acquired immediately nasal of the optic nerve head and covered a retinal area of approximately $30^{\circ} \times 25^{\circ}$. B-scans (16 in total) were averaged at each location using the instrument's eye tracker. Fluorescein angiography scanning laser ophthalmoscope (FA-SLO) images were acquired simultaneously during SD-OCT imaging to document the location of the SD-OCT raster scan on the fundus. Imaging was performed in both eyes, and images from one eye were selected for analysis based on image quality.

2.3. SD-OCT Segmentation. Our previously reported image segmentation software developed in MATLAB (Mathworks Inc., Natick, MA, USA) was used to identify four retinal cell layer interfaces in the SD-OCT B-scans $[56,61,71]$. Retinal cell layer interfaces were detected using graph theory and dynamic programming, based on a previous publication $[72,73]$. In summary, a graph was generated for each SD-OCT B-scan with edge weights assigned based on the magnitude of the vertical gradients in the image, such that large gradients resulted in small weights. A horizontal path through the graph minimizing the total sum of the weights was obtained by Dijkstra's algorithm. Since retinal cell layer boundaries are typically characterized by large vertical intensity gradients, the path minimizing the weights of the graph defined a line separating two distinct retinal cell layers $[72,73]$.

An example of a SD-OCT B-Scan image acquired in a wild-type mouse is shown in Figure 1(a). As labeled, the following individual retinal layers were visualized: combined nerve fiber layer (NFL) and ganglion cell layer (GCL), inner plexiform layer (IPL), inner nuclear layer (INL), outer plexiform layer (OPL), outer nuclear layer (ONL), external limiting membrane (ELM), photoreceptor inner segments (IS), photoreceptor inner segment ellipsoid (ISe), photoreceptor outer segments (OS), retinal pigment epithelium (RPE), and choroid. As shown in Figure 1(b), using a dedicated image segmentation method, four retinal layer interfaces were identified, namely, interfaces between (1) vitreous and internal limiting membrane (ILM), (2) INL and OPL, (3) ONL and ISe, and (4) OS and RPE.

Image segmentation was performed in a set order to find a unique path for these four retinal layer interfaces. First, the vitreous/ILM interface was identified since this was characterized by the largest positive vertical gradient (dark to bright transition) in the image and represented the lowest weighted path. Second, the OS/RPE boundary was found by restricting the graph search area to include only image regions below the vitreous/ILM path. Third, the INL/OPL cell interface was detected by limiting the graph to include only image regions 


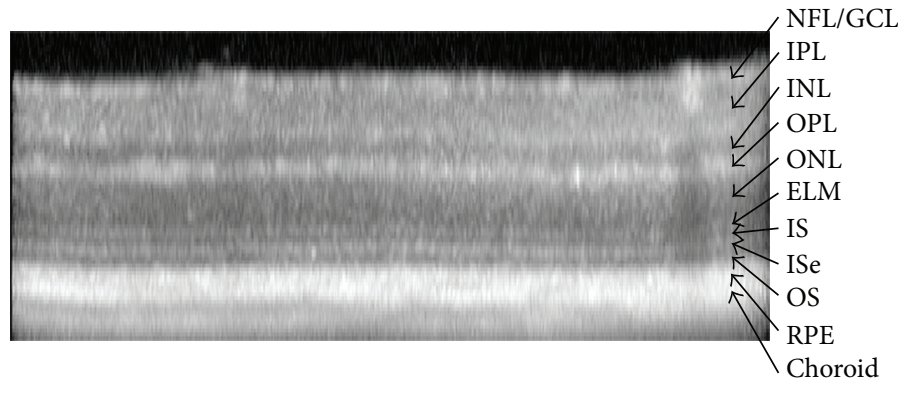

(a)

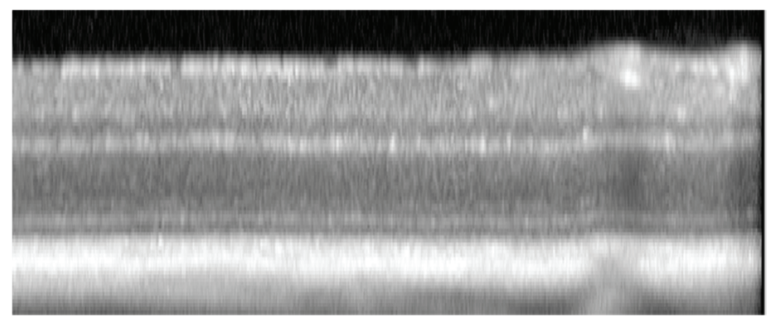

(c)

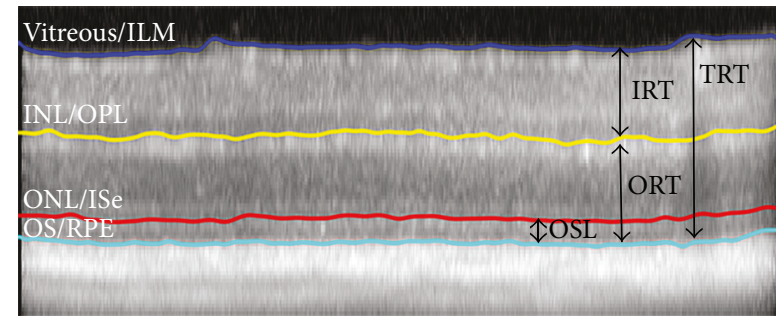

(b)

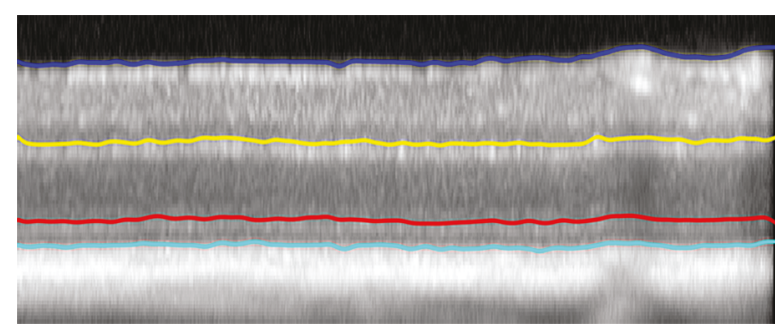

(d)

FIGURE 1: Examples of SD-OCT images acquired in wild-type ( $\mathrm{a}$ and b) and Akita ( $\mathrm{c}$ and $\mathrm{d}$ ) mice. Individual retinal layers are labeled: nerve fiber layer (NFL), ganglion cell layer (GCL), inner plexiform layer (IPL), inner nuclear layer (INL), outer plexiform layer (OPL), outer nuclear layer (ONL), external limiting membrane (ELM), inner segments (IS), inner segment ellipsoid (ISe), outer segments (OS), retinal pigment epithelium (RPE), and choroid. Segmentation lines outlining 4 retinal cell layer interfaces are displayed, corresponding to the vitreous/ internal limiting membrane (ILM); INL/OPL; ONL/ISe; and OS/RPE. Inner retinal thickness (IRT), outer retinal thickness (ORT), total retinal thickness (TRT), and photoreceptor outer segment length (OSL) are indicated by vertical arrows.

between the vitreous/ILM and OS/RPE paths. Finally, the ONL/ISe interface was found by restricting the graph search area to include only image areas between the detected INL/ OPL and OS/RPE interface.

If required, the operator was able to manually correct errors in the detected interfaces in the 31 SD-OCT B-scans. To correct segmentation errors, the operator would select a segmentation path that required modification and manually draw a revised line. The search area of the graph was then restricted to include only a small vertical image region around the manually drawn line, and a revised path for the cell layer interface was obtained by determining a new graph cut solution.

2.4. Thickness Mapping. Inner retinal thickness (IRT), outer retinal thickness (ORT), total retinal thickness (TRT), and photoreceptor outer segment length (OSL) were calculated from each SD-OCT B-scan, as indicated in Figure 1(b). IRT was defined as the depth separation between the vitreous/ ILM and INL/OPL interfaces (IRT $=\mathrm{NFL}+\mathrm{GCL}+\mathrm{IPL}$ + INL). ORT was defined as the depth separation between the INL/OPL and OS/RPE interfaces $(\mathrm{ORT}=\mathrm{OPL}+\mathrm{ONL}$ $+\mathrm{IS}+\mathrm{ISe}+\mathrm{OS}$ ). TRT was determined as the depth separation between the vitreous/ILM and OS/RPE interfaces $(\mathrm{TRT}=\mathrm{IRT}+\mathrm{ORT})$. The OS/RPE interface was used as the retinal posterior boundary (instead of Bruch's membrane) due to the lack of a clear discrimination between the RPE and choroid in all mice. OSL was defined as the depth separation between the ONL/ISe and OS/RPE interfaces $(\mathrm{OSL}=\mathrm{ISe}+\mathrm{OS})$. By compiling thickness data from all SDOCT B-scans, IRT, ORT, TRT, and OSL maps were generated. In each thickness map, measurements that were in close vicinity to the optic disk ( $<1.5$ disk diameter horizontally and vertically from optic disk center) were eliminated to avoid including alterations due to the normal optic disk depression.

2.5. Data Analysis. Global thickness measures (IRT, ORT, TRT, and OSL) were derived by averaging measurements within corresponding thickness maps. A two-way analysis of variance was used to evaluate the effects of condition (Akita and wild-type) and age (12 and 24 weeks) on mean thickness measures and body weight. In some diabetic mice, blood sugar measurements exceeded $600 \mathrm{mg} / \mathrm{dL}$, the maximum level detectable by the glucometer. In these cases, a stand-in blood glucose value of $600 \mathrm{mg} / \mathrm{dL}$ was used and a nonparametric Mann-Whitney rank-sum test was performed to compare blood glucose between diabetic and wild-type mice. Statistical significance was accepted at $p<0.05$.

Local thickness alterations in Akita mice were determined by assigning Z-scores to each pixel location in thickness maps based on the mean and standard deviation (SD) of thickness measurements calculated at the corresponding location from data in all wild-type mice. From each thickness map, the percentage of retinal area with Z-score values $<-1,-1$ to 1 , or $>1$ was calculated. These percentages were averaged among all Akita mice, yielding a distribution that summarized the relative retinal area with thickness values below $1 \mathrm{SD}$ (Z-score $<-1)$, within $1 \mathrm{SD}(-1>\mathrm{Z}$-score $<1)$, and more than 1 SD $(Z$-score $>1)$ compared to normative values of wild-type mice. Using this method, we were able to determine the average percentage of retinal area in Akita mice that 
TABLE 1: Blood glucose measurements of Akita and wild-type mice at 12 and 24 weeks of age.

\begin{tabular}{lcccc}
\hline Variable & Age (weeks) & Akita & Wild-type & $p$ value \\
\hline \multirow{2}{*}{ Blood glucose $(\mathrm{mg} / \mathrm{dL})$} & 12 & $\geq 269$ & $151 \pm 19$ & $<0.001^{*}$ \\
& 24 & $\geq 380$ & $139 \pm 19$ & $<0.001^{*}$ \\
\hline
\end{tabular}

${ }^{*}$ Mann-Whitney rank-sum test.

was thinner $(Z$-score $<-1)$, thicker $(Z$-score $>1)$, or similar $(-1<Z$-score $<1)$ to mean thickness in wild-type mice.

\section{Results}

Blood glucose measurements in Akita and wild-type mice are summarized in Table 1. In wild-type mice, blood glucose measurements were $151 \pm 19 \mathrm{mg} / \mathrm{dL}$ (mean \pm standard deviation, $N=12)$ at 12 weeks and $139 \pm 19 \mathrm{mg} / \mathrm{dL}(N=10)$ at 24 weeks of age. Blood glucose measurements in Akita mice were $269 \mathrm{mg} / \mathrm{dL}(N=10)$ at 12 weeks and $380 \mathrm{mg} / \mathrm{dL}(N=$ $12)$ at 24 weeks of age. There was a significant difference in blood glucose levels between Akita and wild-type mice at both age groups $(p<0.001)$.

Mean body weight of wild-type mice was $28 \pm 3$ and $32 \pm 3$ grams at 12 and 24 weeks of age, respectively. Mean body weight of Akita mice was $24 \pm 2$ grams at both 12 weeks and 24 weeks of age. There was a significant age by condition interaction on body weight $(p=0.04)$. Wild-type mice had greater body weights at 24 weeks compared to 12 weeks of age $(p=0.008)$, while Akita mice had similar body weights at 12 and 24 weeks of age $(p=0.7)$. At both age groups, Akita mice had lower body weight than wild-type mice $(p<0.001)$.

The relative brightness of individual retinal layers in SDOCT images in mice can be visualized in Figures 1(a) and 1(c), similar to normal reflectance properties in humans. In both Akita and wild-type mice, the NFL + GCL, IPL, OPL, ELM, ISe, and RPE were brighter than the INL, ONL, IS, and OS. Additionally, in the inner retina, both IPL and INL were distinctly visualized, while the NFL and GCL appeared as a single combined layer, in agreement with previous reports in mice $[74,75]$. Similarly, interfaces between all outer retinal layers were clearly demarcated, except the interface between RPE and Bruch's membrane. As shown in Figures 1(b) and 1(d), the image segmentation method successfully detected retinal layer interfaces in both wildtype and Akita mice as outlined on SD-OCT images.

Examples of IRT, ORT, TRT, and OSL thickness maps in one Akita and one wild-type mouse each are shown in Figure 2. These maps are visual color representations of variations in retinal thickness based on retinal geography for each separate layer. Areas with increased thickness are represented with warmer colors while areas with decreased thickness are represented with cooler colors. In both mice, major retinal vessels were visualized in the IRT and TRT maps. In addition, IRT, ORT, and TRT maps were higher near the optic disc in both wild-type and Akita mice, while the OSL maps were relatively uniform. Overall, decreased IRT, ORT, and TRT were present in maps of the Akita mouse compared to the wild-type mouse.
The percentage of retinal areas plotted as a function of Zscore for IRT, ORT, TRT, and OSL is shown in Figure 3. The information represented in Figure 3 is a numerical interpretation of the information in Figure 2 with the Z-score as a calculated normative value of the mean. The percentage of retinal area with normal IRT, ORT, TRT, and OSL $(-1<\mathrm{Z}$ score $<1$ ) was $68 \%, 56 \%, 57 \%$, and $68 \%$, respectively. The percentage of retinal area with reduced IRT, ORT, TRT, and OSL (Z-score $<-1$ ) was $27 \%, 38 \%, 41 \%$, and $11 \%$, respectively. The percentage of retinal area with increased IRT, ORT, TRT, and OSL (Z-score >1) was 5\%, 6\%, 3\%, and $21 \%$, respectively. The difference between the percentage of retinal areas with reduced and increased IRT, ORT, and TRT was $22 \%, 32 \%$, and $38 \%$, respectively, indicating that in areas of abnormal retinal thickness, thinning occurred more frequently than thickening.

Compiled numerical IRT, ORT, TRT, and OSL data from all Akita and wild-type mice at 12 and 24 weeks of age are displayed in Table 2 . In the wild-type mouse, the mean of IRT, ORT, and TRT was $94 \pm 4 \mu \mathrm{m}, 114 \pm 3 \mu \mathrm{m}$, and 208 $\pm 6 \mu \mathrm{m}$, respectively, at 12 weeks and $96 \pm 6 \mu \mathrm{m}, 115 \pm 2 \mu \mathrm{m}$, and $211 \pm 7 \mu \mathrm{m}$, respectively, at 24 weeks. In the Akita mouse, the mean of IRT, ORT, and TRT was $92 \pm 3 \mu \mathrm{m}, 112 \pm 5 \mu \mathrm{m}$, and $203 \pm 8 \mu \mathrm{m}$, respectively, at 12 weeks and $92 \pm 4 \mu \mathrm{m}$, $112 \pm 2 \mu \mathrm{m}$, and $204 \pm 5 \mu \mathrm{m}$, respectively, at 24 weeks. Mean OSL in both Akita and wild-type mice was $26 \pm 2 \mu \mathrm{m}$ at 12 weeks, and $27 \pm 2$ and $26 \pm 2$, respectively, at 24 weeks. There was no significant effect of age or interaction between age and condition on IRT, ORT, TRT, or OSL $(p \geq 0.1)$. There was a significant effect of condition on IRT, ORT, and TRT. These thickness measures were significantly thinner in Akita mice compared to wild-type mice $(p \leq 0.02)$, while OSL was similar $(p=0.3)$.

\section{Discussion}

In this study, global and local alterations in retinal layer thickness were compared between Akita and wild-type mice by semiautomated segmentation of SD-OCT images obtained using a commercially available instrument. We report significant global thinning of the total retina due to a reduction in both inner and outer retinal layer thickness in Akita mice at 12 and 24 weeks of age and no significant alterations in photoreceptor outer segment length. Correspondingly, assessment of local thickness alterations showed thinning of inner and outer retinal layers in approximately one third of the evaluated retinal area. These findings indicate that significant neurodegeneration occurs in retinal cell layers of Akita mice as a result of sustained hyperglycemia. The Akita mouse holds promise as a suitable experimental surrogate to study the effects of uncontrolled hyperglycemia on retinal neurodegeneration that often precedes clinically identifiable DR.

In this study, retinal layer thickness was measured in Akita and wild-type mice by SD-OCT imaging, which has become an indispensable tool in the diagnosis and management of DR. Employing SD-OCT imaging allows longitudinal analysis of retinal thickness alterations, a clear advantage over histologic sectioning of retinal tissue. 


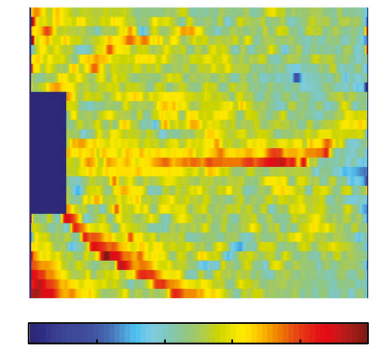

$\begin{array}{lllllll}70 & 80 & 90 & 100 & 110 & 120 & 50\end{array}$
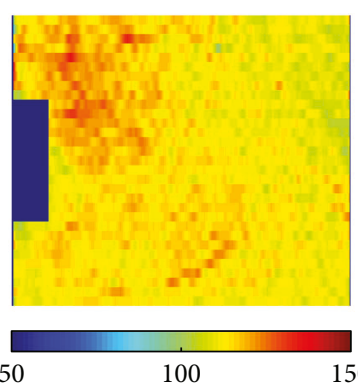

100
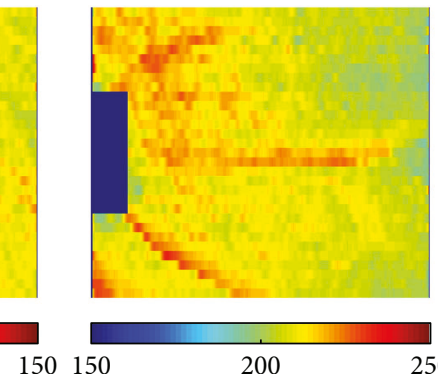

200

(a)
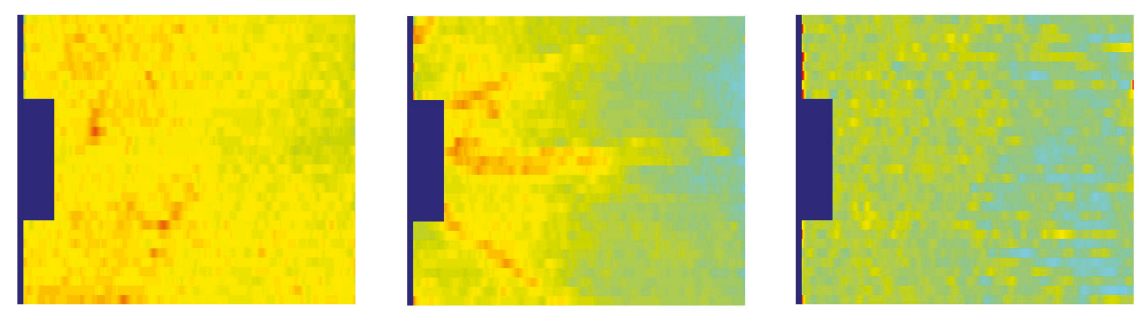

150150

(b)

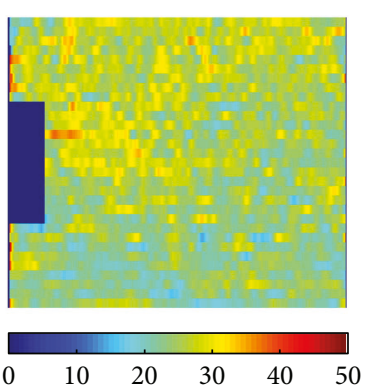

50

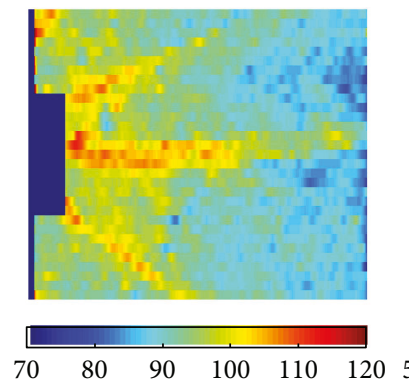

50
100

FIGURE 2: Thickness maps generated in (a) wild-type and (b) Akita mice. Left to right: inner retinal thickness (IRT), outer retinal thickness (ORT), total retinal thickness (TRT), and photoreceptor outer segment length (OSL). Color bars display thickness in microns.

Additionally, in vivo evaluation of retinal thickness using SD-OCT imaging eliminates errors due to tissue shrinkage or damage that may occur during the processing of histologic sections [37]. Overall, SD-OCT imaging is a superior modality for in vivo retinal thickness assessment and is likely more sensitive and specific than ex vivo methods.

Measurements of retinal layer thickness in wild-type mice in the current study were consistent with previous reported values using SD-OCT. TRT, measured using the OS/RPE interface as the posterior retinal boundary, was similar to a previous study that used the same retinal interfaces [76] and in general agreement with previously reported TRT measurements that included RPE thickness [37, 74, 76-78]. Likewise, IRT and ORT in the current study were consistent with the previous studies $[76,79]$ and in general agreement with reported IRT measurements that included OPL thickness $[74,80]$. Overall, the consistency between measurements in the current study and previously published values in wild-type mice serves as validation of retinal layer thickness obtained by our semiautomated image segmentation technique.

In the present study, TRT was reduced in Akita mice due to significant thinning of both inner and outer retinal cell layers. Previous studies have shown reductions in IRT and loss of ganglion cells in Akita mice using histopathology techniques [10, 42, 43, 45, 68]. Barber et al. [10] reported

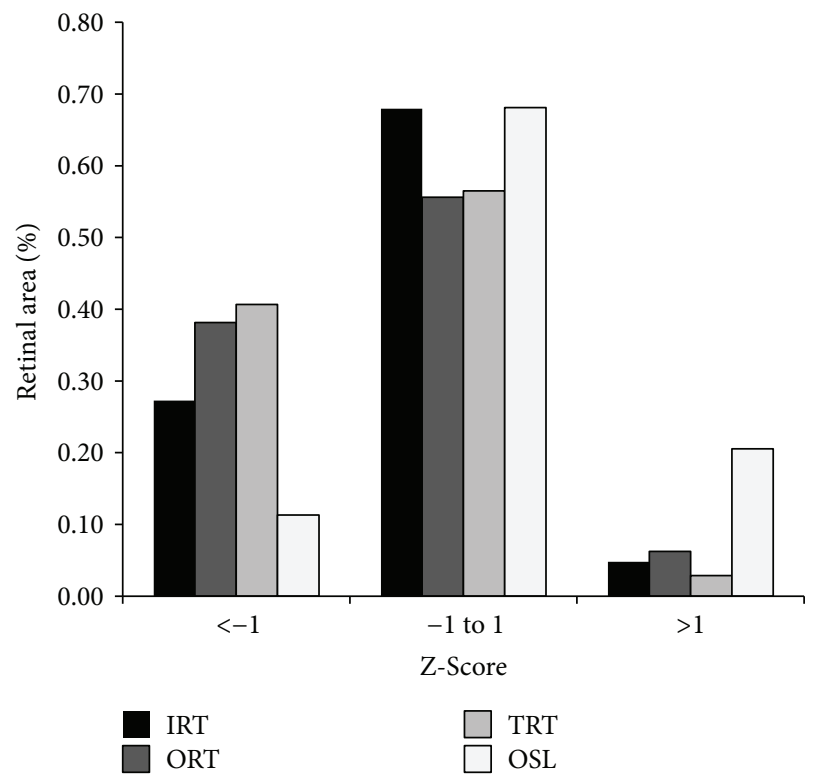

FIgURE 3: The percentage of retinal area plotted as a function of $\mathrm{Z}$ score in Akita mice for inner retinal thickness (IRT), outer retinal thickness (ORT), total retinal thickness (TRT), and photoreceptor outer segment length (OSL). 
TABLE 2: Inner retinal thickness (IRT), outer retinal thickness (ORT), total retinal thickness (TRT), and photoreceptor outer segment length (OSL) in Akita and wild-type mice at 12 and 24 weeks of age.

\begin{tabular}{|c|c|c|c|c|c|c|c|}
\hline \multirow{2}{*}{ Variable (microns) } & \multirow{2}{*}{ Age (weeks) } & \multirow{2}{*}{ Akita } & \multirow{2}{*}{ Wild-type } & \multirow{2}{*}{$\%$ difference } & \multicolumn{3}{|c|}{$p$ value } \\
\hline & & & & & Condition & Age & Condition $*$ age \\
\hline \multirow{2}{*}{ IRT } & 12 & $92 \pm 3$ & $94 \pm 4$ & -2.13 & \multirow{2}{*}{0.02} & \multirow{2}{*}{0.4} & \multirow{2}{*}{0.8} \\
\hline & 24 & $92 \pm 4$ & $96 \pm 6$ & -4.17 & & & \\
\hline \multirow{2}{*}{ ORT } & 12 & $112 \pm 5$ & $114 \pm 3$ & -1.75 & \multirow{2}{*}{0.008} & \multirow{2}{*}{0.3} & \multirow{2}{*}{0.4} \\
\hline & 24 & $112 \pm 2$ & $115 \pm 2$ & -2.61 & & & \\
\hline \multirow{2}{*}{ TRT } & 12 & $203 \pm 8$ & $208 \pm 6$ & -2.40 & \multirow{2}{*}{0.004} & \multirow{2}{*}{0.3} & \multirow{2}{*}{0.6} \\
\hline & 24 & $204 \pm 5$ & $211 \pm 7$ & -3.32 & & & \\
\hline \multirow{2}{*}{ OSL } & 12 & $26 \pm 2$ & $26 \pm 2$ & 0.00 & \multirow{2}{*}{0.3} & \multirow{2}{*}{0.1} & \multirow{2}{*}{1.0} \\
\hline & 24 & $27 \pm 2$ & $26 \pm 2$ & +3.85 & & & \\
\hline
\end{tabular}

significant thinning of the IPL and INL as well as a reduction in the number of ganglion cell bodies in Akita mice compared to wild-type mice at 22 weeks of age. Smith et al. [81] reported that by 17 to 25 weeks of age, Akita mice demonstrated a $30 \%$ reduction in the IPL thickness, a $25 \%$ decrease in INL thickness, and a 30\% reduction in ganglion cells. Rakoczy et al. [43] also reported significant thinning of the ganglion cell layer in Akita mice compared to wild-type mice. Our finding of reduced IRT and ORT by in vivo imaging of Akita mice is in agreement with a previous study that used a similar imaging modality [33].

Other studies utilizing SD-OCT imaging have reported no differences in TRT between Akita and wild-type mice $[37,70]$. The discrepancy between these findings may be attributed to variations in sample size, imaging region, and image segmentation method. Since identification of Bruch's membrane is more challenging in mice than in humans, the detection of this interface with the use of commercially available automated segmentation software may be less accurate and more variable compared to the current technique which used the OS/RPE boundary for calculation of TRT.

Clinical studies in human type 1 diabetics with minimal or no retinopathy report retinal thinning, similar to the findings in the Akita mouse, suggesting this model may be a suitable surrogate for the study of neurodegenerative alterations in the retina that precede clinically identifiable DR $[12,13$, 17, 63, 82-84]. Biallosterski et al. [49] compared retinal thickness measurements of patients with and without type 1 diabetes mellitus and found that the mean retinal thickness in the pericentral area was thinner in diabetic patients. Asefzadeh et al. [48] reported that the degree of macular and foveal thinning correlated with duration of disease in diabetic subjects with minimal or no DR. Additionally, van Dijk et al. $[12,64]$ found significant thinning of the inner retinal layers, specifically the GCL, IPL, and INL, in type 1 diabetic patients with minimal retinopathy as compared to healthy controls. Taken collectively, these findings indicate that neurodegenerative alterations may cause thinning of the inner retinal layers, a potential hallmark of subclinical DR. If true, the ability of the Akita mouse to reproduce reduced retinal thickness validates its use as an appropriate model to study early neurodegenerative changes in the human retina.
The etiology of retinal thickness changes reported in this study is likely due to vascular, inflammatory, degenerative, and neural abnormalities that occur as a result of sustained hyperglycemia. Vascular incompetency demonstrated by acellular capillaries, decreased choroidal blood flow, imbalanced pro- and antiangiogenic factors including vascular endothelial growth factor, and leakage has been reported in this model $[31,36,41,42]$. Inflammatory changes, including the presence of reactive microglial cells, were shown to be present as early as eight weeks of age [10]. Degenerative changes including pericyte ghosts, basement membrane thickening, decreased a- and b-wave amplitudes, dendrite remodeling, and increased apoptosis of retinal ganglion cells have been previously reported $[10,17,32,36,69]$. In addition, neural abnormalities such as morphological alterations in astrocytes, decreased cholinergic and dopaminergic amacrine cells, and increased inflammatory leukocytes have also been observed in this model [10, 17, 68, 69]. Further in vivo studies utilizing high-resolution imaging systems are needed to determine the interplay of these factors that contribute to decreased retinal thickness in this experimental model.

\section{Conclusion}

In summary, significant thinning of inner, outer, and total retinal thickness was reported in the Ins2 (Akita) diabetic mouse using SD-OCT imaging. These findings support the use of the Akita mouse as an experimental model to study the effects of hyperglycemia on retinal thickness.

\section{Disclosure}

The funders had no role in the design and conduct of the study; collection, management, analysis, and interpretation of the data; preparation, review, or approval of the manuscript; and decision to submit the manuscript for publication.

\section{Conflicts of Interest}

The authors declare that they have no conflicts of interest. 


\section{Acknowledgments}

This study was supported by Illinois Society for the Prevention of Blindness (ISPB), National Institutes of Health Research Grants EY017918 and EY001792, Senior Scientific Investigator (Mahnaz Shahidi), and unrestricted departmental awards from Research to Prevent Blindness.

\section{References}

[1] C. C. Cowie, K. F. Rust, D. D. Byrd-Holt et al., "Prevalence of diabetes and impaired fasting glucose in adults in the U.S. population: National Health And Nutrition Examination Survey 1999-2002," Diabetes Care, vol. 29, no. 6, pp. 1263-1268, 2006.

[2] C. C. Cowie, K. F. Rust, E. S. Ford et al., "Full accounting of diabetes and pre-diabetes in the U.S. population in 19881994 and 2005-2006," Diabetes Care, vol. 32, no. 2, pp. 287294, 2009.

[3] D. Pascolini and S. P. Mariotti, "Global estimates of visual impairment: 2010," British Journal of Ophthalmology, vol. 96, no. 5, pp. 614-618, 2012.

[4] S. Wild, G. Roglic, A. Green, R. Sicree, and H. King, "Global prevalence of diabetes: estimates for the year 2000 and projections for 2030," Diabetes Care, vol. 27, no. 5, pp. 1047-1053, 2004.

[5] G. Tremolada, C. Del Turco, R. Lattanzio et al., "The role of angiogenesis in the development of proliferative diabetic retinopathy: impact of intravitreal anti-VEGF treatment," Experimental Diabetes Research, vol. 2012, Article ID 728325, 8 pages, 2012.

[6] J. W. Miller, J. Le Couter, E. C. Strauss, and N. Ferrara, "Vascular endothelial growth factor a in intraocular vascular disease," Ophthalmology, vol. 120, no. 1, pp. 106-114, 2013.

[7] A. Das and P. G. McGuire, "Retinal and choroidal angiogenesis: pathophysiology and strategies for inhibition," Progress in Retinal and Eye Research, vol. 22, no. 6, pp. 721-748, 2003.

[8] R. F. Gariano, D. Hu, and J. Helms, "Expression of angiogenesis-related genes during retinal development," Gene Expression Patterns, vol. 6, no. 2, pp. 187-192, 2006.

[9] R. F. Gariano and T. W. Gardner, "Retinal angiogenesis in development and disease," Nature, vol. 438, no. 7070, pp. 960-966, 2005.

[10] A. J. Barber, D. A. Antonetti, T. S. Kern et al., "The Ins2 $2^{\text {Akita }}$ mouse as a model of early retinal complications in diabetes," Investigative Ophthalmology \& Visual Science, vol. 46, no. 6, pp. 2210-2218, 2005.

[11] T. N. Crawford, D. V. Alfaro, J. B. Kerrison, and E. P. Jablon, "Diabetic retinopathy and angiogenesis," Current Diabetes Reviews, vol. 5, no. 1, pp. 8-13, 2009.

[12] H. W. van Dijk, P. H. Kok, M. Garvin et al., "Selective loss of inner retinal layer thickness in type 1 diabetic patients with minimal diabetic retinopathy," Investigative Ophthalmology \& Visual Science, vol. 50, no. 7, pp. 3404-3409, 2009.

[13] H. W. van Dijk, F. D. Verbraak, P. H. Kok et al., "Early neurodegeneration in the retina of type 2 diabetic patients," Investigative Ophthalmology \& Visual Science, vol. 53, no. 6, pp. 2715-2719, 2012.

[14] F. de Oliveira, "Pericytes in diabetic retinopathy," British Journal of Ophthalmology, vol. 50, no. 3, pp. 134-143, 1966.
[15] D. J. Addison, A. Garner, and N. Ashton, "Degeneration of intramural pericytes in diabetic retinopathy," $B M J$, vol. 1, no. 5691, pp. 264-266, 1970.

[16] A. M. Abu El-Asrar, L. Dralands, L. Missotten, and K. Geboes, "Expression of antiapoptotic and proapoptotic molecules in diabetic retinas," Eye, vol. 21, no. 2, pp. 238-245, 2007.

[17] A. J. Barber, E. Lieth, S. A. Khin, D. A. Antonetti, A. G. Buchanan, and T. W. Gardner, "Neural apoptosis in the retina during experimental and human diabetes. Early onset and effect of insulin," Journal of Clinical Investigation, vol. 102, no. 4, pp. 783-791, 1998.

[18] E. Chihara, T. Matsuoka, Y. Ogura, and M. Matsumura, "Retinal nerve fiber layer defect as an early manifestation of diabetic retinopathy," Ophthalmology, vol. 100, no. 8, pp. 11471151, 1993.

[19] K. J. Hellgren, B. Bengtsson, and E. Agardh, "Functional and structural change in diabetic eyes. Interim results from an ongoing longitudinal prospective study," Acta Ophthalmologica, vol. 91, no. 7, pp. 672-677, 2013.

[20] H. Takahashi, T. Goto, T. Shoji, M. Tanito, M. Park, and E. Chihara, "Diabetes-associated retinal nerve fiber damage evaluated with scanning laser polarimetry," American Journal of Ophthalmology, vol. 142, no. 1, pp. 88-94, 2006.

[21] S. F. Abcouwer and T. W. Gardner, "Diabetic retinopathy: loss of neuroretinal adaptation to the diabetic metabolic environment," Annals of the New York Academy of Sciences, vol. 1311, no. 1no. 1, pp. 174-190, 2014.

[22] P. Speiser, A. M. Gittelsohn, and A. Patz, "Studies on diabetic retinopathy: III. Influence of diabetes on intramural pericytes," Archives of Ophthalmology, vol. 80, no. 3, pp. 332-337, 1968.

[23] E. Lieth, A. J. Barber, B. Xu et al., "Glial reactivity and impaired glutamate metabolism in short-term experimental diabetic retinopathy. Penn State Retina Research Group," Diabetes, vol. 47, no. 5, pp. 815-820, 1998.

[24] F. Bandello, R. Lattanzio, I. Zucchiatti, and C. Del Turco, "Pathophysiology and treatment of diabetic retinopathy," Acta Diabetologica, vol. 50, no. 1, pp. 1-20, 2013.

[25] J. M. Kezic, X. Chen, E. P. Rakoczy, and P. G. McMenamin, "The effects of age and $\mathrm{Cx}_{3} \mathrm{crl}$ deficiency on retinal microglia in the Ins $2^{\text {Akita }}$ diabetic mouse," Investigative Ophthalmology \& Visual Science, vol. 54, no. 1, pp. 854-863, 2013.

[26] P. Z. Costa and R. Soares, "Neovascularization in diabetes and its complications. Unraveling the angiogenic paradox," Life Sciences, vol. 92, no. 22, pp. 1037-1045, 2013.

[27] D. S. McLeod, G. A. Lutty, S. D. Wajer, and R. W. Flower, "Visualization of a developing vasculature," Microvascular Research, vol. 33, no. 2, pp. 257-269, 1987.

[28] R. Casey and W. W. Li, "Factors controlling ocular angiogenesis," American Journal of Ophthalmology, vol. 124, no. 4, pp. 521-529, 1997.

[29] P. A. D'Amore, "Mechanisms of retinal and choroidal neovascularization," Investigative Ophthalmology \& Visual Science, vol. 35, no. 12, pp. 3974-3979, 1994.

[30] D. T. Shima, A. P. Adamis, N. Ferrara et al., "Hypoxic induction of endothelial cell growth factors in retinal cells: identification and characterization of vascular endothelial growth factor (VEGF) as the mitogen," Molecular Medicine, vol. 1, no. 2, pp. 182-193, 1995.

[31] S. McLenachan, A. L. Magno, D. Ramos et al., "Angiography reveals novel features of the retinal vasculature in healthy 
and diabetic mice," Experimental Eye Research, vol. 138, pp. 621,2015

[32] C. Guo, Z. Zhang, P. Zhang et al., "Novel transgenic mouse models develop retinal changes associated with early diabetic retinopathy similar to those observed in rats with diabetes mellitus," Experimental Eye Research, vol. 119, pp. 77-87, 2014.

[33] J. R. Hombrebueno, M. Chen, R. G. Penalva, and H. Xu, "Loss of synaptic connectivity, particularly in second order neurons is a key feature of diabetic retinal neuropathy in the Ins $2^{\text {Akita }}$ mouse," PLoS One, vol. 9, no. 5, article e97970, 2014.

[34] I. Soto, G. R. Howell, C. W. John, J. L. Kief, R. T. Libby, and S. W. John, "DBA/2J mice are susceptible to diabetic nephropathy and diabetic exacerbation of IOP elevation," PLoS One, vol. 9, no. 9, article e107291, 2014.

[35] J. Wisniewska-Kruk, I. Klaassen, I. M. Vogels et al., "Molecular analysis of blood-retinal barrier loss in the Akimba mouse, a model of advanced diabetic retinopathy," Experimental Eye Research, vol. 122, pp. 123-131, 2014.

[36] Z. Han, J. Guo, S. M. Conley, and M. I. Naash, "Retinal angiogenesis in the Ins $2^{\text {Akita }}$ mouse model of diabetic retinopathy," Investigative Ophthalmology \& Visual Science, vol. 54, no. 1, pp. 574-584, 2013.

[37] S. McLenachan, X. Chen, P. G. McMenamin, and E. P. Rakoczy, "Absence of clinical correlates of diabetic retinopathy in the Ins $2^{\text {Akita }}$ retina," Clinical \& Experimental Ophthalmology, vol. 41, no. 6, pp. 582-592, 2013.

[38] N. P. Akimov and R. C. Rentería, "Spatial frequency threshold and contrast sensitivity of an optomotor behavior are impaired in the Ins $2^{\text {Akita }}$ mouse model of diabetes," Behavioural Brain Research, vol. 226, no. 2, pp. 601-605, 2012.

[39] P. F. Kador, P. Zhang, J. Makita et al., "Novel diabetic mouse models as tools for investigating diabetic retinopathy," PLoS One, vol. 7, no. 12, article e49422, 2012.

[40] Y. Liu, L. F. Leo, C. McGregor, A. Grivitishvili, C. J. Barnstable, and J. Tombran-Tink, "Pigment epithelium-derived factor (PEDF) peptide eye drops reduce inflammation, cell death and vascular leakage in diabetic retinopathy in Ins $2^{\text {Akita }}$ mice," Molecular Medicine, vol. 18, pp. 1387-1401, 2012.

[41] E. R. Muir, R. C. Rentería, and T. Q. Duong, "Reduced ocular blood flow as an early indicator of diabetic retinopathy in a mouse model of diabetes," Investigative Ophthalmology \& Visual Science, vol. 53, no. 10, pp. 6488-6494, 2012.

[42] W. S. Wright, A. S. Yadav, R. M. McElhatten, and N. R. Harris, "Retinal blood flow abnormalities following six months of hyperglycemia in the Ins2(Akita) mouse," Experimental Eye Research, vol. 98, pp. 9-15, 2012.

[43] E. P. Rakoczy, I. S. Ali Rahman, N. Binz et al., "Characterization of a mouse model of hyperglycemia and retinal neovascularization," The American Journal of Pathology, vol. 177, no. 5, pp. 2659-2670, 2010.

[44] L. Cheng, X. Han, and Y. Shi, “A regulatory role of LPCAT1 in the synthesis of inflammatory lipids, PAF and LPC, in the retina of diabetic mice," American Journal of Physiology Endocrinology and Metabolism, vol. 297, no. 6, pp. E1276-E1282, 2009.

[45] W. M. Freeman, G. V. Bixler, R. M. Brucklacher et al., “Transcriptomic comparison of the retina in two mouse models of diabetes," Journal of Ocular Biology, Diseases, and Informatics, vol. 2, no. 4, pp. 202-213, 2009.
[46] M. Adhi and J. S. Duker, "Optical coherence tomography current and future applications," Current Opinion in Ophthalmology, vol. 24, no. 3, pp. 213-221, 2013.

[47] T. Alasil, P. A. Keane, J. F. Updike et al., "Relationship between optical coherence tomography retinal parameters and visual acuity in diabetic macular edema," Ophthalmology, vol. 117, no. 12 , pp. 2379-2386, 2010.

[48] B. Asefzadeh, B. M. Fisch, C. E. Parenteau, and A. A. Cavallerano, "Macular thickness and systemic markers for diabetes in individuals with no or mild diabetic retinopathy," Clinical \& Experimental Ophthalmology, vol. 36, no. 5, pp. 455-463, 2008.

[49] C. Biallosterski, M. E. van Velthoven, R. P. Michels, R. O. Schlingemann, J. H. DeVries, and F. D. Verbraak, "Decreased optical coherence tomography-measured pericentral retinal thickness in patients with diabetes mellitus type 1 with minimal diabetic retinopathy," British Journal of Ophthalmology, vol. 91, no. 9, pp. 1135-1138, 2007.

[50] K. V. Chalam, S. B. Bressler, A. R. Edwards et al., "Retinal thickness in people with diabetes and minimal or no diabetic retinopathy: Heidelberg Spectralis optical coherence tomography," Investigative Ophthalmology \& Visual Science, vol. 53, no. 13, pp. 8154-8161, 2012.

[51] F. Forooghian, P. F. Stetson, S. A. Meyer et al., "Relationship between photoreceptor outer segment length and visual acuity in diabetic macular edema," Retina, vol. 30, no. 1, pp. 63-70, 2010.

[52] R. Hua, L. Liu, X. Wang, and L. Chen, "Imaging evidence of diabetic choroidopathy in vivo: angiographic pathoanatomy and choroidal-enhanced depth imaging," PLoS One, vol. 8, no. 12, article e83494, 2013.

[53] B. Y. Kim, S. D. Smith, and P. K. Kaiser, "Optical coherence tomographic patterns of diabetic macular edema," American Journal of Ophthalmology, vol. 142, no. 3, pp. 405-412.e1, 2006.

[54] J. I. Lim, R. Zelkha, M. Niec, V. Setlur, and M. Shahidi, "Inner and outer retinal thickness mapping of nonproliferative diabetic retinopathy by spectral-domain optical coherence tomography," Ophthalmic Surgery, Lasers and Imaging Retina, vol. 46, no. 3, pp. 316-320, 2015.

[55] A. S. Maheshwary, S. F. Oster, R. M. Yuson, L. Cheng, F. Mojana, and W. R. Freeman, "The association between percent disruption of the photoreceptor inner segment-outer segment junction and visual acuity in diabetic macular edema," American Journal of Ophthalmology, vol. 150, no. 1, pp. 63-67.e1, 2010.

[56] F. Mohammad, R. Ansari, J. Wanek, A. Francis, and M. Shahidi, "Feasibility of level-set analysis of enface OCT retinal images in diabetic retinopathy," Biomedical Optics Express, vol. 6, no. 5, pp. 1904-1918, 2015.

[57] T. Murakami, K. Nishijima, T. Akagi et al., "Optical coherence tomographic reflectivity of photoreceptors beneath cystoid spaces in diabetic macular edema," Investigative Ophthalmology \& Visual Science, vol. 53, no. 3, pp. 1506-1511, 2012.

[58] H. J. Shin, S. H. Lee, H. Chung, and H. C. Kim, “Association between photoreceptor integrity and visual outcome in diabetic macular edema," Graefe's Archive for Clinical and Experimental Ophthalmology, vol. 250, no. 1, pp. 61-70, 2012.

[59] B. L. Sikorski, G. Malukiewicz, J. Stafiej, H. Lesiewska-Junk, and D. Raczynska, "The diagnostic function of OCT in diabetic maculopathy," Mediators of Inflammation, vol. 2013, Article ID 434560, 12 pages, 2013. 
[60] E. H. Sohn, J. J. Chen, K. Lee, M. Niemeijer, M. Sonka, and M. D. Abràmoff, "Reproducibility of diabetic macular edema estimates from SD-OCT is affected by the choice of image analysis algorithm," Investigative Ophthalmology \& Visual Science, vol. 54, no. 6, pp. 4184-4188, 2013.

[61] A. W. Francis, J. Wanek, J. I. Lim, and M. Shahidi, "Enface thickness mapping and reflectance imaging of retinal layers in diabetic retinopathy," PLoS One, vol. 10, no. 12, article e0145628, 2015.

[62] M. Esmaeelpour, S. Brunner, S. Ansari-Shahrezaei et al., "Choroidal thinning in diabetes type 1 detected by 3-dimensional $1060 \mathrm{~nm}$ optical coherence tomography," Investigative Ophthalmology \& Visual Science, vol. 53, no. 11, pp. 6803-6809, 2012.

[63] J. M. Lopes de Faria, H. Russ, and V. P. Costa, "Retinal nerve fibre layer loss in patients with type 1 diabetes mellitus without retinopathy," British Journal of Ophthalmology, vol. 86, no. 7, pp. 725-728, 2002.

[64] H. W. van Dijk, F. D. Verbraak, P. H. Kok et al., "Decreased retinal ganglion cell layer thickness in patients with type 1 diabetes," Investigative Ophthalmology \& Visual Science, vol. 51, no. 7, pp. 3660-3665, 2010.

[65] K. W. Bronson-Castain, M. A. Bearse, J. Neuville et al., “Adolescents with type 2 diabetes: early indications of focal retinal neuropathy, retinal thinning, and venular dilation," Retina, vol. 29, no. 5, pp. 618-626, 2009.

[66] C. V. Regatieri, L. Branchini, J. Carmody, J. G. Fujimoto, and J. S. Duker, "Choroidal thickness in patients with diabetic retinopathy analyzed by spectral-domain optical coherence tomography," Retina, vol. 32, no. 3, pp. 563-568, 2012.

[67] M. S. Islam, "Animal models of diabetic neuropathy: progress since 1960s," Journal of Diabetes Research, vol. 2013, Article ID 149452, 9 pages, 2013.

[68] M. J. Gastinger, R. S. Singh, and A. J. Barber, "Loss of cholinergic and dopaminergic amacrine cells in streptozotocindiabetic rat and Ins $2^{\text {Akita }}$-diabetic mouse retinas," Investigative Ophthalmology \& Visual Science, vol. 47, no. 7, pp. 3143-3150, 2006.

[69] M. J. Gastinger, A. R. Kunselman, E. E. Conboy, S. K. Bronson, and A. J. Barber, "Dendrite remodeling and other abnormalities in the retinal ganglion cells of Ins $2^{\text {Akita }}$ diabetic mice," Investigative Ophthalmology \& Visual Science, vol. 49, no. 6, pp. 2635-2642, 2008.

[70] N. N. Vagaja, N. Binz, S. McLenachan, E. P. Rakoczy, and P. G. McMenamin, "Influence of endotoxin-mediated retinal inflammation on phenotype of diabetic retinopathy in $I n s 2^{A-}$ kita mice," British Journal of Ophthalmology, vol. 97, no. 10, pp. 1343-1350, 2013.

[71] J. Wanek, R. Zelkha, J. I. Lim, and M. Shahidi, "Feasibility of a method for en face imaging of photoreceptor cell integrity," American Journal of Ophthalmology, vol. 152, no. 5, pp. 807-814.e1, 2011.

[72] S. J. Chiu, X. T. Li, P. Nicholas, C. A. Toth, J. A. Izatt, and S. Farsiu, "Automatic segmentation of seven retinal layers in SDOCT images congruent with expert manual segmentation," Optics Express, vol. 18, no. 18, pp. 19413-19428, 2010.

[73] S. J. Chiu, C. A. Toth, C. Bowes Rickman, J. A. Izatt, and S. Farsiu, "Automatic segmentation of closed-contour features in ophthalmic images using graph theory and dynamic programming," Biomedical Optics Express, vol. 3, no. 5, pp. 1127-1140, 2012.
[74] B. J. Antony, W. Jeong, M. D. Abramoff, J. Vance, E. H. Sohn, and M. K. Garvin, "Automated 3D segmentation of intraretinal surfaces in SD-OCT volumes in normal and diabetic mice," Translational Vision Science \& Technology, vol. 3, no. 5, p. 8, 2014.

[75] C. Dysli, V. Enzmann, R. Sznitman, and M. S. Zinkernagel, "Quantitative analysis of mouse retinal layers using automated segmentation of spectral domain optical coherence tomography images," Translational Vision Science \& Technology, vol. 4, no. 4, p. 9, 2015.

[76] L. R. Ferguson, J. M. Dominguez 2nd, S. Balaiya, S. Grover, and K. V. Chalam, "Retinal thickness normative data in wild-type mice using customized miniature SD-OCT," PLoS One, vol. 8, no. 6, article e67265, 2013.

[77] G. Huber, S. C. Beck, C. Grimm et al., "Spectral domain optical coherence tomography in mouse models of retinal degeneration," Investigative Ophthalmology \& Visual Science, vol. 50, no. 12, pp. 5888-5895, 2009.

[78] M. Ruggeri, H. Wehbe, S. Jiao et al., "In vivo three-dimensional high-resolution imaging of rodent retina with spectral-domain optical coherence tomography," Investigative Ophthalmology \& Visual Science, vol. 48, no. 4, pp. 1808-1814, 2007.

[79] L. R. Ferguson, S. Grover, J. M. Dominguez II, S. Balaiya, and K. V. Chalam, "Retinal thickness measurement obtained with spectral domain optical coherence tomography assisted optical biopsy accurately correlates with ex vivo histology," PLoS One, vol. 9, no. 10, article e111203, 2014.

[80] M. K. Aziz, A. Ni, D. A. Esserman, and S. H. Chavala, "Evidence of early ultrastructural photoreceptor abnormalities in light-induced retinal degeneration using spectral domain optical coherence tomography," British Journal of Ophthalmology, vol. 98, no. 7, pp. 984-989, 2014.

[81] S. B. Smith, J. Duplantier, Y. Dun et al., "In vivo protection against retinal neurodegeneration by sigma receptor 1 ligand (+)-pentazocine," Investigative Ophthalmology \& Visual Science, vol. 49, no. 9, pp. 4154-4161, 2008.

[82] A. J. Barber, "A new view of diabetic retinopathy: a neurodegenerative disease of the eye," Progress in NeuroPsychopharmacology and Biological Psychiatry, vol. 27, no. 2, pp. 283-290, 2003.

[83] E. L. Fletcher, J. A. Phipps, and J. L. Wilkinson-Berka, "Dysfunction of retinal neurons and glia during diabetes," Clinical and Experimental Optometry, vol. 88, no. 3, pp. 132-145, 2005.

[84] E. Lieth, T. W. Gardner, A. J. Barber, D. A. Antonetti, and Group PSRR, "Retinal neurodegeneration: early pathology in diabetes," Clinical \& Experimental Ophthalmology, vol. 28, no. 1, pp. 3-8, 2000. 


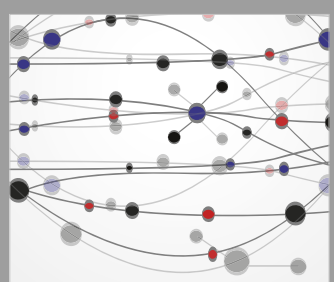

The Scientific World Journal
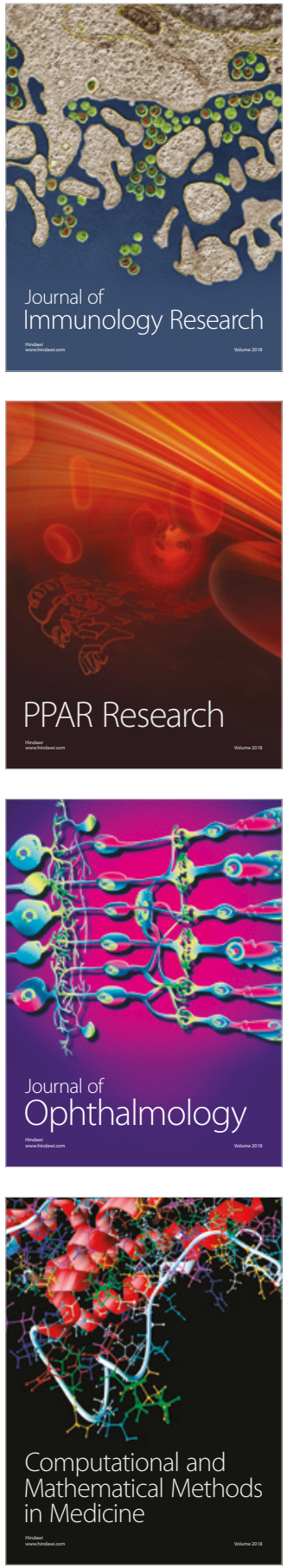

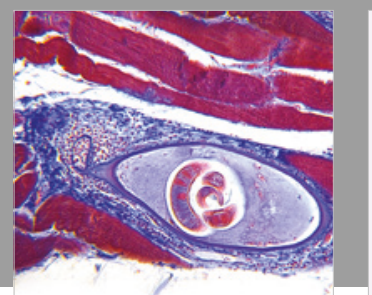

Gastroenterology Research and Practice

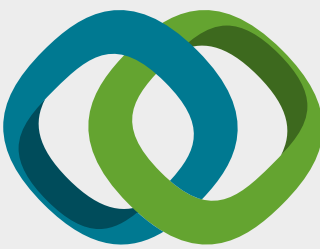

\section{Hindawi}

Submit your manuscripts at

www.hindawi.com
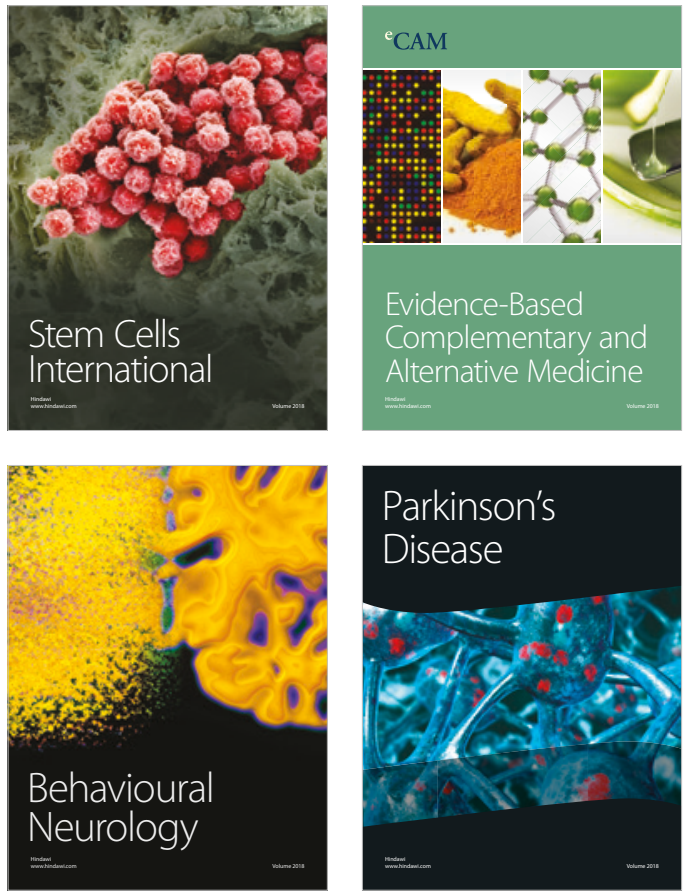

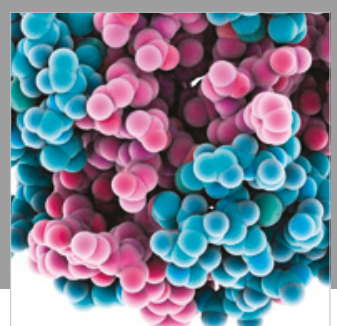

ournal of

Diabetes Research

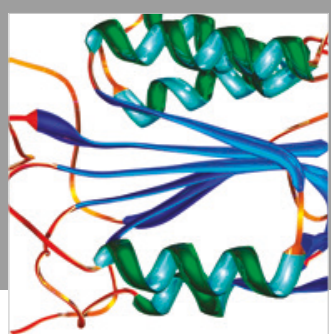

Disease Markers
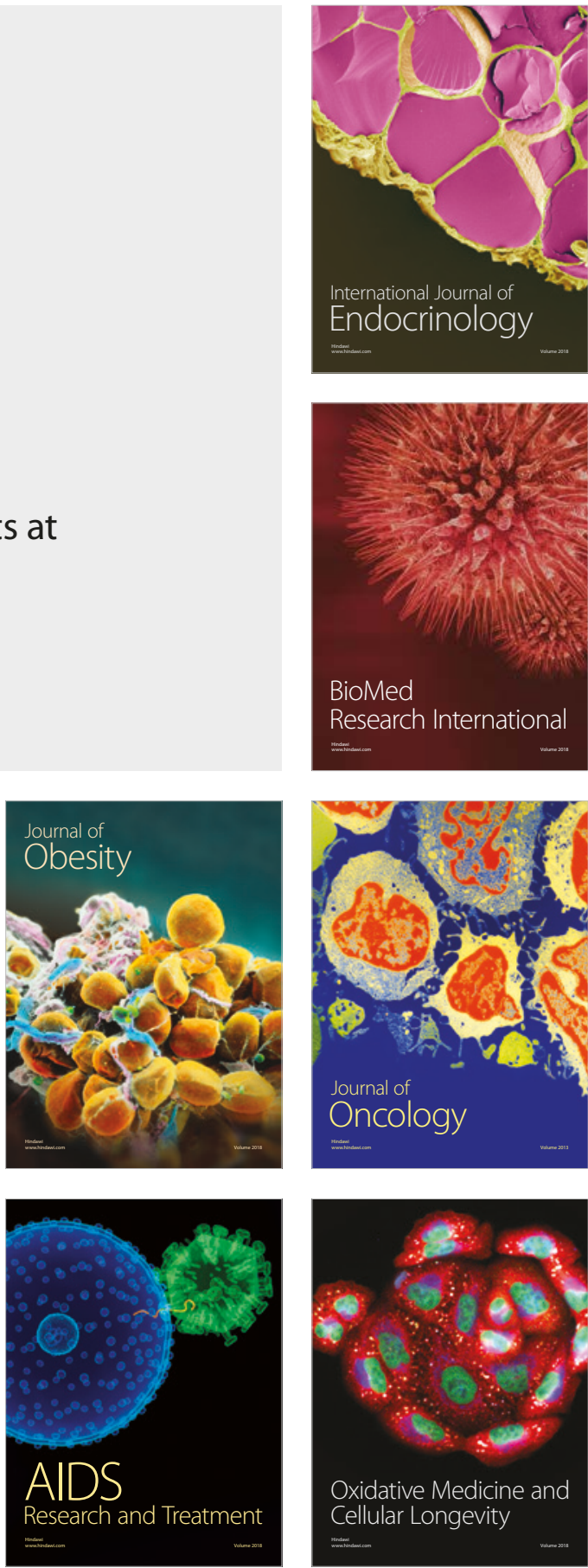